Europhysics Letters

PREPRINT

\title{
Modeling planar degenerate wetting and anchoring in nematic liquid crystals
}

\author{
J.-B. Fournier ${ }^{1,2}$ and P. Galatola ${ }^{1}$ \\ 1 Matière et Systèmes Complexes, UMR 7057 CNRS \& Université Paris 7 - 2 place \\ Jussieu, F-75251 Paris Cedex 05, France \\ 2 Laboratoire de Physico-Chimie Théorique, UMR 7083 CNRS-ESPCI, 10 rue Vauquelin, \\ F-75231 Paris Cedex 05, France
}

PACS. 61.30.Hn - Liquid crystals: surface phenomena.

PACS. $61.30 . \mathrm{Dk}$ - Continuum models and theories of liquid crystal structure.

PACS. 64.70.Md - Transitions in liquid crystals.

\begin{abstract}
We propose a simple surface potential describing the planar degenerate anchoring of nematic liquid crystals, i.e., the tendency of the molecules to align parallel to one another along any direction parallel to the surface. We show that, at lowest order in the tensorial Landau-de Gennes order-parameter, fourth-order terms must be included. We analyze the anchoring and wetting properties of this surface potential. In the nematic phase, we find the desired degenerate planar anchoring, with positive scalar order-parameter and some surface biaxiality. In the isotropic phase, we find, in agreement with experiments, that the wetting layer may exhibit a uniaxial ordering with negative scalar order-parameter. For large enough anchoring strength, this negative ordering transits towards the planar degenerate state.
\end{abstract}

Introduction. - Nematic liquid crystals are non-polar liquids consisting of elongated molecules aligned, on the average, parallel to a common direction $\pm \boldsymbol{n}$ [1]. The unit vector $\boldsymbol{n}$ is called the director. Exceptionally, in the case of "negative order," the molecules may be distributed perpendicularly to $\boldsymbol{n}$. The nematic order is quantified by a symmetric traceless tensor $\mathrm{Q}$, of Cartesian components $Q_{i j}(i, j=1,2,3)$. Microscopically, calling $\boldsymbol{m}$ a unit vector parallel to the long axis of the molecules, the tensor order-parameter can be defined as $Q_{i j}=\left\langle m_{i} m_{j}\right\rangle-\frac{1}{3} \delta_{i j}$, where the brackets denote statistical averaging and $\delta_{i j}$ is the Kronecker symbol. For uniaxial ordering, which corresponds to the preferred state of the nematic in the bulk, two of the eigenvalues of Q are equal; in this case $Q_{i j}$ can be written as $S\left(n_{i} n_{j}-\frac{1}{3} \delta_{i j}\right)$, where $-\frac{1}{2} \leq S \leq 1$ is the scalar order-parameter. Note that $S=0$ corresponds to the isotropic phase (randomly oriented molecules), occurring at temperatures above the nematic-isotropic phase transition; $S>0$ corresponds to the ordinary nematic, in which the molecules are aligned on the average parallel to $\boldsymbol{n}$, while $S<0$ corresponds to the "negative" case, in which the molecules are on the average orthogonal to $\boldsymbol{n}$.

The alignment of a nematic liquid crystal by a bounding interface is of considerable interest both for fundamental and technological reasons. In the most common situations, this "anchoring" effect is well described by a surface free energy depending only on the director

(c) EDP Sciences 
$\boldsymbol{n}$ at the interface [1]. Three typical situations are: i) homeotropic anchoring, where the preferred, or "easy", average orientation corresponds to $\boldsymbol{n}$ normal to the interface, ii) planar anchoring, where the preferred average orientation corresponds to $\boldsymbol{n}$ lying in one particular direction parallel to the interface, and iii) planar degenerate anchoring, where all the planar orientations for $\boldsymbol{n}$ are equivalent easy directions. The latter situation is often realized with isotropic bounding fluids, e.g., glycerin [2] or gallium [3]; degenerate anchorings can also be obtained with polymer-grafted surfaces [4].

In general, surface interactions also perturb the degree of nematic ordering, e.g., the value of $S$. Above the bulk nematic-isotropic transition temperature, this effect may induce the wetting of the surface by a nematic layer with $S \neq 0$, as predicted by Sheng [5]. This effect, long ago observed by Miyano [6], was also recently detected by a surface force apparatus measuring the related capillary condensation [7]. Although nematics are uniaxial in the bulk, the wetting layer may exhibit biaxiality due to the lower symmetry near the surface [8]. In the biaxial case, the symmetric and traceless $Q_{i j}$ has its three eigenvalues $\lambda_{i}(i=1,2,3)$ all different. A nematic director, $\boldsymbol{n}$, can still be defined as the eigenvector corresponding to the eigenvalue of largest modulus, say $\lambda_{3}$. By analogy with the uniaxial case, one can then define the scalar order-parameter by $\lambda_{3}=\frac{2}{3} S$; the amount of biaxiality, $0 \leq B \leq 1$, is then defined by $\lambda_{1}=-\frac{1}{3} S(1-B)$ and $\lambda_{2}=-\frac{1}{3} S(1+B)$.

The aim of the present paper is to model the planar degenerate anchoring of nematic liquid crystals, using the tensorial Landau-de Gennes formalism. To our knowledge, this has not been done before. Actually, there is some confusion in the literature, since a number of papers dealing with "degenerate planar anchoring" describe in fact homeotropic anchoring with negative $S$ [8-11]. The confusion arises from the fact that the molecules themselves are planar and randomly oriented in this homeotropic situation. Again, conventional planar degenerate anchoring describes the tendency of a substrate to favor the common alignment of the molecules along any direction parallel to its surface [1]. This is the very situation that we consider in this paper.

Theory. - Let us recall some of the existing tensorial anchoring models. The simplest quadratic surface free energy favoring a given non-degenerate nematic ordering $Q^{(0)}$, i.e., a well-defined director and a well-defined scalar and, possibly, biaxial order-parameter, was proposed by Nobili and Durand [12]:

$$
f_{\mathrm{ND}}=\frac{1}{2} W\left(Q_{i j}-Q_{i j}^{(0)}\right)\left(Q_{i j}-Q_{i j}^{(0)}\right) .
$$

Here, and throughout, summation over repeated indices is implicit. Because $f_{\mathrm{ND}}$ is proportional to the sum of the squares of the components of $\mathrm{Q}-\mathrm{Q}^{(0)}$, it obviously has, for $W>0$, a unique minimum for $Q=Q^{(0)}$. In this simple situation, the surface energy itself possesses a well-defined minimum. In principle, this is not necessary: the only requirement is that the nematic free energy as a whole, i.e., the sum of the surface and the bulk free energies, be bounded from below. For instance, early descriptions have dealt with only a linear surface free energy $\nu_{i} Q_{i j} \nu_{j}$, where $\boldsymbol{\nu}$ is the normal to the interface [5]. Actually, it is not clear at which order the surface free energy expansion should be truncated. In principle, for any truncation, one should verify that the higher-order terms are indeed negligible (this is especially critical in the case of large surface couplings). Another difficulty, when dealing with truncated surface free energies not bounded from below, is that one cannot determine which kind of anchoring they favor without including bulk terms and solving the whole problem. For these reasons, we shall concentrate in this paper on surface free energies that possess well-defined minima by themselves. 


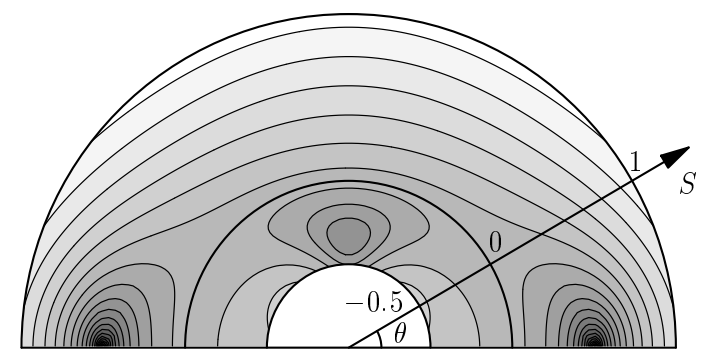

Fig. 1 - Polar contour plot of the surface potential (4), with $W_{1}=W_{2}$ and $S_{0}=0.5$, for a uniaxial surface order-parameter $Q_{i j}=S\left(n_{i} n_{j}-\frac{1}{3} \delta_{i j}\right)$, where $\boldsymbol{n}=(\cos \theta \cos \phi, \cos \theta \sin \phi, \sin \theta)$. The angle $\theta$ is the inclination of the director with respect to the substrate and the radial coordinate corresponds to the scalar order-parameter $-\frac{1}{2} \leq S \leq 1$. The energy does not depend on $\phi$. Darker shadings corresponds to lower energies. The absolute minimum is attained for degenerate planar anchoring: $\theta=0$ (or $\pi$ ) and $S=S_{0}$; the apparent homeotropic minimum for $\theta=\pi / 2$ and $S<0$ is actually a saddle-point with respect to the biaxial degree of freedom (outside the plane of this figure).

The most general quadratic form describing isotropic substrates is $[9,13]$

$$
f_{2}=W_{11} \nu_{i} Q_{i j} \nu_{j}+W_{21} Q_{i j} Q_{i j}+W_{22}\left(\nu_{i} Q_{i j} \nu_{j}\right)^{2}+W_{23} \nu_{i} Q_{i j} Q_{j k} \nu_{k} .
$$

It contains all the scalars up to quadratic order made with $Q$ and $\boldsymbol{\nu}$ (the surface normal). Minimizing $f_{2}$ with respect to the five components of the most general symmetric traceless tensor yields, in the basis where the $z$-axis is along $\boldsymbol{\nu}$, a unique extremum [14], uniaxial, at $\mathrm{Q}^{(0)}=\operatorname{diag}(\lambda, \lambda,-2 \lambda)$, where $\lambda=W_{11} /\left(4 W_{22}+4 W_{23}+6 W_{21}\right)$. This corresponds to a homeotropic anchoring $(\boldsymbol{n}=\boldsymbol{\nu})$ with either $S>0$ or $S<0$ depending on the sign of $\lambda$.

To obtain a planar degenerate uniaxial minimum, it is necessary to include terms of order higher than two. We shall go up to fourth order to have a surface free energy bounded from below. Note that this is also the lowest order for the bulk free energy to correctly describe the nematic-isotropic phase transition. Our aim is to obtain a degenerate manifold of minima for $Q_{i j}=S_{0}\left(n_{i} n_{j}-\frac{1}{3} \delta_{i j}\right)$, where $\boldsymbol{n}$ is an arbitrary unit vector parallel to the substrate, and where $S_{0}>0$ is fixed. To this purpose, we define

$$
\tilde{Q}_{i j}=Q_{i j}+\frac{1}{3} S_{0} \delta_{i j}
$$

and we look for the constraints ensuring that $\tilde{Q}_{i j}$ takes the form $S_{0} n_{i} n_{j}$, where $\boldsymbol{n}$ is parallel to the substrate. The two following conditions are necessary and sufficient [15]: $i$ ) $\tilde{Q}_{i j}$ must coincide with its projection on the substrate $\tilde{Q}_{i j}^{\perp}=P_{i k} \tilde{Q}_{k \ell} P_{\ell j}$, where $P_{i j}=\delta_{i j}-\nu_{i} \nu_{j}$, and ii) the trace of the square of $\tilde{Q}_{i j}$ must be equal to $S_{0}{ }^{2}$, that is $\tilde{Q}_{i j} \tilde{Q}_{i j}=S_{0}{ }^{2}$. The simplest surface free energy being minimum if, and only if, these two conditions are satisfied is

$$
f_{4}=W_{1}\left(\tilde{Q}_{i j}-\tilde{Q}_{i j}^{\perp}\right)\left(\tilde{Q}_{i j}-\tilde{Q}_{i j}^{\perp}\right)+W_{2}\left(\tilde{Q}_{i j} \tilde{Q}_{i j}-{S_{0}}^{2}\right)^{2},
$$

with $W_{1}>0$ and $W_{2}>0$. This surface potential is of fourth-order in $\mathrm{Q}$ and covariant. It is not, however, the most general fourth-order expansion giving rise to a planar degenerate anchoring: one can add, for instance, a contribution proportional to the square of the first term and several other similar, but linearly independent, terms. In the basis where the $z$-axis 
is along the surface normal $\boldsymbol{\nu}$, the above surface potential takes the explicit form

$$
\begin{aligned}
f_{4} & =W_{1}\left[2\left(Q_{x z}^{2}+Q_{y z}^{2}\right)+\left(Q_{x x}+Q_{y y}-\frac{S_{0}}{3}\right)^{2}\right] \\
& +4 W_{2}\left(Q_{x x}^{2}+Q_{y y}^{2}+Q_{x x} Q_{y y}+Q_{x y}^{2}+Q_{x z}^{2}+Q_{y z}^{2}-\frac{S_{0}^{2}}{3}\right)^{2},
\end{aligned}
$$

where we have taken into account that $\mathrm{Q}$ is symmetric and traceless $\left(Q_{z z}=-Q_{x x}-Q_{y y}\right)$. As shown in fig. 11 the surface potential $f_{4}$ possesses a degenerate absolute minimum for uniaxial order-parameters with planar director and $S=S_{0}>0$. No other minima are present: the apparent homeotropic minimum with negative scalar order-parameter visible in fig. [1] is actually a saddle point with respect to biaxial perturbations.

Wetting and anchoring properties. - To test the physical properties of the surface potential (4), we consider a semi-infinite sample filled, for $z>0$, with a liquid crystal either in the nematic or in the isotropic phase. For the bulk free energy density we take the fourth-order Landau-de Gennes expansion [1]:

$$
f_{V}=\frac{1}{2} a\left(T-T^{\star}\right) Q_{i j} Q_{i j}-\frac{1}{3} b Q_{i j} Q_{j k} Q_{k i}+\frac{1}{4} c\left(Q_{i j} Q_{i j}\right)^{2}+\frac{1}{2} L Q_{i j, k} Q_{i j, k},
$$

where the comma indicates spatial derivation. The coefficients $a, b, c$ are assumed positive, $L>0$ is an elastic constant (in the one constant approximation), $T$ is the temperature, and $T^{\star}$ is the supercooling temperature of the isotropic phase. We define the normalized temperature $\tau=27 a c b^{-2}\left(T-T^{\star}\right)$ and the rescaled order-parameter $q_{i j}=\frac{3}{2} \sqrt{6} b^{-1} c Q_{i j}$. The first-order nematic-isotropic transition occurs for $\tau=1$. In the nematic phase $(\tau<1)$, the reduced scalar order-parameter is $s_{b}(\tau)=\frac{1}{4} \sqrt{3 / 2}(3+\sqrt{9-8 \tau})$. We rescale the lengths with respect to the correlation length at the transition $\xi=b^{-1} \sqrt{27 c L}$ and the energies with respect to $F_{0}=$ $2 b[L /(3 c)]^{3 / 2}$. The dimensionless bulk and surface free energy densities become $\frac{1}{2} \tau q_{i j} q_{i j}-$ $\sqrt{6} q_{i j} q_{j k} q_{k i}+\frac{1}{2}\left(q_{i j} q_{i j}\right)^{2}+\frac{1}{2} q_{i j, k} q_{i j, k}$ and $w_{1}\left(\tilde{q}_{i j}-\tilde{q}_{i j}^{\perp}\right)\left(\tilde{q}_{i j}-\tilde{q}_{i j}^{\perp}\right)+w_{2}\left(\tilde{q}_{i j} \tilde{q}_{i j}-s_{0}^{2}\right)^{2}$, respectively, with the normalized anchoring parameters $w_{1}=3 b^{-1}(3 c / L)^{1 / 2} W_{1}, w_{2}=\frac{2}{3} b\left(3 L c^{3}\right)^{-1 / 2} W_{2}$ and $s_{0}=\frac{3}{2} \sqrt{6} b^{-1} c S_{0}$. We look for equilibrium order-parameter profiles depending only on $z$ and possessing a symmetry plane orthogonal to the substrate, which we take as the $(y, z)$ plane. Therefore, we set

$$
\mathrm{q}(z)=\left(\begin{array}{ccc}
-\alpha(z)-\beta(z) & 0 & 0 \\
0 & \alpha(z) & \gamma(z) \\
0 & \gamma(z) & \beta(z)
\end{array}\right)
$$

The minimization problem defined by $f_{V}$ and $f_{4}$ yields the Euler-Lagrange equations in the bulk:

$$
\begin{aligned}
& \frac{d^{2} \alpha}{d z^{2}}=\tau \alpha-\sqrt{6}\left(\alpha^{2}-2 \beta^{2}+\gamma^{2}-2 \alpha \beta\right)+4 \alpha\left(\alpha^{2}+\beta^{2}+\gamma^{2}+\alpha \beta\right) \\
& \frac{d^{2} \beta}{d z^{2}}=\tau \beta-\sqrt{6}\left(\beta^{2}-2 \alpha^{2}+\gamma^{2}-2 \alpha \beta\right)+4 \beta\left(\alpha^{2}+\beta^{2}+\gamma^{2}+\alpha \beta\right), \\
& \frac{d^{2} \gamma}{d z^{2}}=\gamma\left[\tau-3 \sqrt{6}(\alpha+\beta)+4\left(\alpha^{2}+\beta^{2}+\gamma^{2}+\alpha \beta\right)\right],
\end{aligned}
$$

with the boundary conditions on the substrate, i.e., at $z=0$,

$$
\frac{d \alpha}{d z}=\frac{8}{3} w_{2} \alpha\left[3\left(\alpha^{2}+\beta^{2}+\gamma^{2}+\alpha \beta\right)-s_{0}^{2}\right]-\frac{2}{9} w_{1}\left(3 \beta+s_{0}\right),
$$




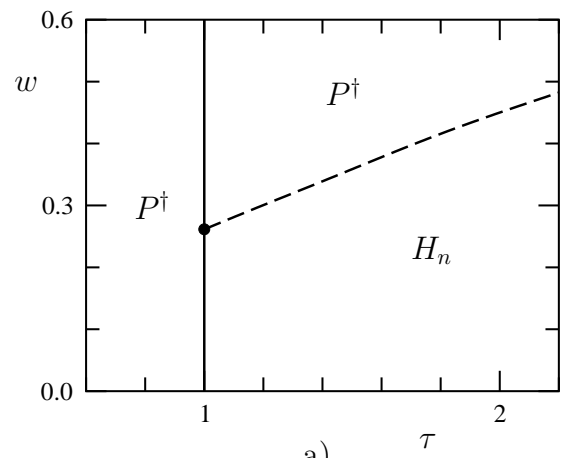

a)

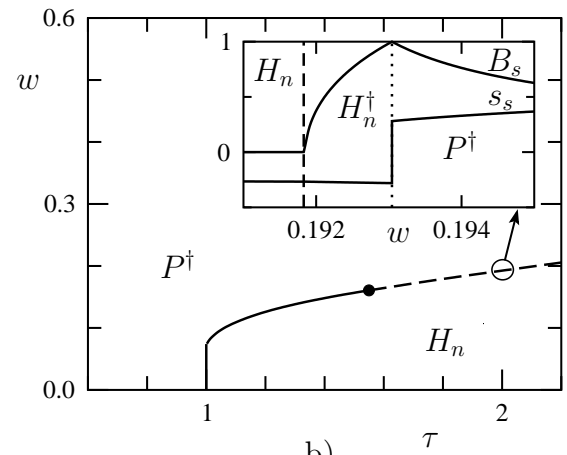

b)

Fig. 2 - a) Phase diagram of the surface state in the plane of the reduced temperature $\tau$ and of the reduced anchoring strength $w=w_{1}=w_{2}$, for the preferred surface scalar order-parameter $s_{0}=1<s_{b}(1)$. The states $P^{\dagger}$ and $H_{n}$ correspond to a planar biaxial ordering with positive scalar order-parameter and to a homeotropic uniaxial ordering with negative scalar order-parameter, respectively. Full (resp. dashed) lines indicate first (resp. second) order transitions. The second-order line terminates at a critical end-point. b) Same as a) but for $s_{0}=1.5>s_{b}(1)$. The dot indicates a tricritical point. Inset: Evolution of the reduced surface scalar order-parameter, $s_{s}$, and of the amount of biaxiality, $B_{s}$, as a function of $w$, close to the second-order transition at $\tau=2$. The latter, indicated by the dashed vertical line, actually occurs between $H_{n}$ and a homeotropic negative biaxial state $H_{n}^{\dagger}$. At the maximum of biaxiality (dotted vertical line), the state $H_{n}^{\dagger}$ transforms continuously into the state $P^{\dagger}$, the two phases having the same symmetry.

$$
\begin{aligned}
& \frac{d \beta}{d z}=\frac{8}{3} w_{2} \beta\left[3\left(\alpha^{2}+\beta^{2}+\gamma^{2}+\alpha \beta\right)-s_{0}^{2}\right]+\frac{4}{9} w_{1}\left(3 \beta+s_{0}\right) \\
& \frac{d \gamma}{d z}=\gamma\left[\frac{8}{3} w_{2}\left[3\left(\alpha^{2}+\beta^{2}+\gamma^{2}+\alpha \beta\right)-s_{0}^{2}\right]+\frac{6}{3} w_{1}\right]
\end{aligned}
$$

and for $z \rightarrow \infty, d \alpha / d z=d \beta / d z=d \gamma / d z=0$.

We solve numerically the above boundary value problem by a finite difference algorithm with deferred correction and Newton iteration [16]. When multiple solutions are present, we select the one with the lowest total free energy. For the sake of simplicity, we set $w_{1}=w_{2}=w$ and, for a given value of $s_{0}$, we explore the behavior of the order-parameter profile for different values of $\tau$ and $w$. We find that the eigenvectors of the order-parameter solutions are always parallel to the $x, y$ and $z$ axes, which corresponds to $\gamma(z)=0$.

In the nematic phase $(\tau<1)$, we find the expected planar anchoring, with, however, a slight biaxiality in a surface layer of thickness $\simeq \xi$. The nematic director $\boldsymbol{n}$ is parallel to the surface and uniform along $z$, the scalar order-parameter $s>0$ relaxes over $\simeq \xi$ to the bulk value $s_{b}$ starting from a surface value $s_{s}$ intermediate between $s_{0}$ and $s_{b}$. The amount of biaxiality at the surface, $B_{s}$, vanishes for $s_{0}=s_{b}$ and grows with $\left|s_{0}-s_{b}\right|$; it obviously vanishes for $w=0$, but also for $w \rightarrow \infty$, presenting a maximum at a finite value of $w$.

In the isotropic phase $(\tau>1)$, the order-parameter vanishes in the bulk, but a wetting nematic layer appears at the surface. For large values of $w$, the ordering at the surface is planar and biaxial, as in the nematic phase. We call this state $P^{\dagger}$ (the dagger indicating biaxiality). As before, the amount of biaxiality tends to zero as $w \rightarrow \infty$. Conversely, for small values of $w$, the ordering at the surface is homeotropic and uniaxial, with a negative scalar order-parameter. We call this state $H_{n}$. In between, a surface transition occurs.

In fig. 2] we show the typical surface phase diagrams in the plane $(\tau, w)$ for two different 
values of $s_{0}$. At the bulk phase transition $\tau=1$, for small values of $w$ there is always a first-order transition between the states $P^{\dagger}$ and $H_{n}$. For larger values of $w$, two possible scenarios occur, depending on the value of $s_{0}$ with respect to the bulk value at the transition $s_{b}(1)=\sqrt{3 / 2} \simeq 1.22$. When $s_{0}<s_{b}(1)$ (fig. 27 ) there is a first-order transition between two different $P^{\dagger}$ states. When $s_{0}>s_{b}(1)$ (fig. 2b), no surface transition occurs: approaching $\tau=1$ from above, a wetting nematic layer of diverging thickness continuously invades the whole sample. This corresponds to a complete wetting situation, similar to the uniaxial case described in Ref. [5,6,17]; indeed, the surface-induced biaxiality relaxes over $\simeq \xi$. In the isotropic phase $(\tau>1)$, a surface phase transition occurs at a given value of $w$. For $s_{0}>s_{b}$ and $\tau<\tau_{c}$ (corresponding to the tricritical point in fig. 2 $\mathbf{b}$ ), the transition is of first order, between the states $H_{n}$ and $P^{\dagger}$. For $s_{0}<s_{b}$ (fig. 27 a), and for $\tau>\tau_{c}$ when $s_{0}>s_{b}$ (fig. 2] $\mathrm{b}$ ), the transition is of second order, between $H_{n}$ and a biaxial homeotropic negative state, $H_{n}^{\dagger}$. In fact, by symmetry, it is impossible to go continuously from $H_{n}$ to $P^{\dagger}$, since, when the nematic director switches from one eigenvector to another, the amount of biaxiality must be maximum [the order-parameter tensor is then of the form $\operatorname{diag}(0, \lambda,-\lambda)]$. This behavior is illustrated in the inset of fig. 2]. Note that the transformation from $H_{n}^{\dagger}$ to $P^{\dagger}$ is a continuous evolution between two states having the same symmetry and not a phase transition: the discontinuity in $s_{s}$ and the cusp in $B_{s}$, which occur when the biaxiality is maximum, do not correspond to any actual discontinuity of $\mathrm{q}$. In fact, close to this transformation, the tensor $\mathrm{q}(z)$ at the surface $z=0$ can be written as

$$
q(0)=\left(\begin{array}{ccc}
\epsilon & 0 & 0 \\
0 & \sigma-\epsilon & 0 \\
0 & 0 & -\sigma
\end{array}\right),
$$

with $\sigma>0$ and $\epsilon$ a small quantity $(|\epsilon|<\sigma)$ continuosly varying from positive to negative across the transformation. In the $H_{n}^{\dagger}$ state, $\epsilon>0$ : the eigenvalue of $\mathrm{q}(0)$ of maximum modulus is $-\sigma<0$. This corresponds to a nematic director along $z$ (homeotropic ordering), with the negative scalar order-parameter $s_{s}=-3 \sigma / 2$ and the amount of biaxiality $B_{s}=$ $1-2 \epsilon / \sigma$. In the $P^{\dagger}$ state, $\epsilon<0$ : the eigenvalue of maximum modulus is now $\sigma-\epsilon>0$. This corresponds to a nematic director along $y$ (planar ordering), with the positive scalar order parameter $s_{s}=3(\sigma-\epsilon) / 2$ and the amount of biaxiality $B_{s}=1+2 \epsilon /(\sigma-\epsilon)$. At the transformation, $\epsilon=0$ : no nematic director can be defined and the amount of biaxiality is maximum $\left(B_{s}=1\right)$. Therefore, the discontinuous change of sign of $s_{s}$ and the cusp of $B_{s}$ at the transformation are not due to a discontinuity of $q(0)$ but to the change of the direction associated to the eigenvalue of maximum modulus. Note also that the width of the $H_{n}^{\dagger}$ region is extremely narrow and goes to zero at the tricritical point.

Our findings are compatible with the experimental data of Tarczon and Miyano [17], who observed a pretransitional uniaxial negative birefringence with optical axis perpendicular to the surface, corresponding to our $H_{n}$ state, for the compound MBBA in contact with a silanetreated substrate giving planar degenerate anchoring in the nematic phase. This negative pretransitional birefringence was not observed for the compound 5CB on the same substrate, which probably corresponds to our $P^{\dagger}$ surface state.

Conclusions. - As we have shown, the surface free energy describing a planar degenerate anchoring in the framework of the Landau-de Gennes formalism must be at least of fourthorder in the tensorial order-parameter Q. Here, we have proposed the simplest expression having this property and we have investigated the associated anchoring and wetting behavior. In the nematic phase, we find the expected planar degenerate anchoring with, however, a small surface biaxiality. In the isotropic phase, a surface wetting layer appears: for large enough 
surface couplings it is planar biaxial, while for low couplings it is homeotropic uniaxial with negative scalar order-parameter. These predictions are compatible with the experimental observations for substrates yielding planar degenerate anchoring [17]. The transition between the two surface states may be of first or second order. The biaxiality in the planar states tends to zero for large surface coupling, yielding a uniaxial degenerate planar anchoring or wetting. It would be interesting to study the consequences of this degenerate anchoring in more complicated geometries such as, e.g., colloidal suspensions.

Note added in proofs. - After this work was completed, Prof. Sluckin kindly pointed out to us that the surface potentialof ref. [8], linear in the tensor order-parameter, gives a genuine planar degenerate anchoring in the case of complete wetting. We checked numerically that this is true, although the ordering is strongly biaxial in a coherence length close to the surface. Accordingly, above the transition temperature, where the wetting layer is of thickness comparable to the coherence length, the surface layer is strongly biaxial. Our fourth-order potential, on the other hand, gives an almost perfectly planar ordering in the whole microscopic layer, since it directly favors the planar anchoring.

We thank B. Silva for a critical reading of the manuscript and useful comments.

\section{REFERENCES}

[1] De Gennes P.-G. and Prost J., The Physics of Liquid Crystals (Oxford Science Publications, Oxford) 1993.

[2] Lavrentovich O. D. and Pergamenshchik V. M., Mol. Cryst. Liq. Cryst., 179 (1990) 125.

[3] Warenghem M., Isaert N. and Bernard P., J. Opt, 15 (1984) 133.

[4] Ou Ramdane O. et Al., Phys. Rev. Lett., 84 (2000) 3871.

[5] Sheng P., Phys. Rev. Lett., 37 (1976) 1059; Phys. Rev. A, 26 (1982) 1610.

[6] Miyano K., Phys. Rev. Lett., 43 (1979) 51.

[7] Kočevar K., Borštnik A., Muševič I. and Žumer S., Phys. Rev. Lett., 86 (2001) 5914.

[8] Sluckin T. J. and Poniewierski A., Phys. Rev. Lett., 55 (1985) 2907.

[9] Sen A. K. and Sullivan D. E., Phys. Rev. A, 35 (1987) 1391.

[10] Seidin R., Hornreich R. M. and Allender D. W., Phys. Rev. E, 55 (1997) 4302.

[11] Huber M. and Stark H., Europhys. Lett., 69 (2005) 135

[12] Nobili M. and Durand G., Phys. Rev. A, 46 (1992) R6174.

[13] Sluckin T. J. and Poniewierski A., in Fluid Interfacial Phenomena, edited by Croxton C. A. (Wiley, Chichester) 1986.

[14] Degenerate minima are possible if $W_{21}=0$, or if $W_{21}=-\frac{2}{3}\left(W_{22}+W_{23}\right)$ and $W_{11}=0$. The first case corresponds to any $\mathrm{Q}$ possessing one eigenvector along $\boldsymbol{\nu}$ (hence with arbitrary biaxiality). The second case corresponds to $\mathrm{Q}$ uniaxial homeotropic with arbitrary $S$.

[15] According to the first condition, the non-zero components of $\tilde{Q}_{\tilde{\tau}}$, in the basis where the $z$-axis is along $\boldsymbol{\nu}$, can be written as $\tilde{Q}_{x x}=\lambda, \tilde{Q}_{x y}=\tilde{Q}_{y x}=\mu$ and $\tilde{Q}_{y y}=S_{0}-\lambda$. Then the second condition implies $\mu^{2}=\lambda\left(S_{0}-\lambda\right) \geq 0$. Hence, there exists an angle $\theta$ such that $\lambda=S_{0} \cos ^{2} \theta$ and $\mu=S_{0} \sin \theta \cos \theta$. Thus, $\tilde{Q}_{i j}=S_{0} n_{i} n_{j}$ with $\boldsymbol{n}=(\cos \theta, \sin \theta, 0)$.

[16] Ascher U., Mattheij R. M. M. and Russell R. D., Numerical Solution of Boundary Value Problems for Ordinary Differential Equations (Prentice Hall, Englewood Cliffs, NJ) 1988.

[17] Tarczon J. C. and Miyano K., J. Chem. Phys., 73 (1980) 1994. 